\title{
Numerical Scheme for a Viscous Shallow Water System Including New Friction Laws of Second Order: Validation and Application
}

\author{
Olivier Delestre and Ulrich Razafison
}

\section{Introduction}

Free surface flows are described by the Navier-Stokes equations. It is well-known that the numerical resolution of these equations can be expensive. For this reason the shallow water equations (or Saint-Venant system [1]) are widely used in hydraulics and hydrology to simulate free surface flows. We can refer, for instance, to rain-water overland flows [2-5], river flows [6], flooding [7], dam breaks [8, 9], and also oceanography [10]. These equations are derived from the Navier-Stokes equations under the assumption that the ratio between the vertical and the horizontal scales is small. However, the friction laws that are obtained from this derivation, are not used in hydraulics and hydrology. In this context, the main friction laws used are the ones of Manning and Darcy-Weisbach types.

In this paper, we propose to derive the shallow-water equations with Manning or Darcy-Weisbach friction terms, starting from the Navier-Stokes equations with suitable wall-laws at the bottom boundary. Details of the derivation, which follows the ideas of $[11,12]$, will be presented in a forthcoming work. As an approximation of first order, we obtain the classical Shallow water model (without diffusion source term) with the classical Manning or Darcy-Weisbach laws. We next perform a second order approximation and we obtain a viscous Shallow water model with new friction laws of Manning or Darcy-Weisbach type. We then propose a numerical scheme for the new model which is mainly based on a well-balanced

\footnotetext{
O. Delestre

Laboratory J.A. Dieudonné CNRS UMR 7351 \& Polytech Nice - Sophia,

UNSA, Arequipa, Peru

e-mail: delestre@unice.fr

U. Razafison ( $\bowtie)$

Laboratoire de Mathématiques, CNRS UMR 6623, Université de Franche-Comté,

16 Route de Gray, 25030 Besançon Cedex, France

e-mail: ulrich.razafison@univ-fcomte.fr
} 
finite volume method. Then we present analytical validation of the scheme on steady state reference solutions obtained following the main lines of [13]. Finally, this model is applied on real data.

\section{Derivation of the Model}

We give here the key points of the derivation of the model. Details will be given in a forthcoming work. First, we consider the 2D free surface Navier-Stokes equations

$$
\begin{aligned}
\frac{\partial u}{\partial x}+\frac{\partial w}{\partial z} & =0 \\
\frac{\partial u}{\partial t}+\frac{\partial u^{2}}{\partial x}+\frac{\partial u w}{\partial z}+\frac{\partial p}{\partial x} & =\frac{\partial \sigma_{\mathrm{xx}}}{\partial x}+\frac{\partial \sigma_{\mathrm{xz}}}{\partial z} \\
\frac{\partial w}{\partial t}+\frac{\partial u w}{\partial x}+\frac{\partial w^{2}}{\partial z}+\frac{\partial p}{\partial z} & =-g+\frac{\partial \sigma_{\mathrm{zx}}}{\partial x}+\frac{\partial \sigma_{\mathrm{zz}}}{\partial z}
\end{aligned}
$$

where $u$ and $w$ are respectively the horizontal and the vertical components of the velocity, $p$ is the pressure, $\sigma$ is the viscosity tensor, $Z$ is the topography variation and $g=9.81 \mathrm{~m} / \mathrm{s}^{2}$ is the gravity acceleration.

System (1) needs to be completed with conditions on the free surface and at the bottom. At the bottom $z=Z(x)$, a Manning or a Darcy-Weisbach formula is prescribed as follows

$$
\sigma_{\mathrm{xz}}=\mathrm{k} \frac{|u| u}{h^{\alpha}}
$$

where $\alpha \in(0,1 / 3)$.

If $\alpha=0$ (respectively $\alpha=1 / 3$ ), then a Darcy-Weisbach type formula is obtained with $k=f / 8$ (resp. $k=n^{2} g$ ), where $f$ (resp. $n$ ) is the Darcy-Weisbach (resp. Manning) roughness coefficient. This roughness coefficient depends mainly on the roughness of the soil, some values are tabulated in literature depending on the kind of ground considered [14]. Following the same lines as in [11, 12], the derivation of the Shallow Water system of first order is first obtained

$$
\begin{gathered}
\frac{\partial h}{\partial t}+\frac{\partial h u}{\partial x}=0 \\
\frac{\partial h u}{\partial t}+\frac{\partial h u^{2}}{\partial x}+\frac{g}{2} \frac{\partial h^{2}}{\partial x}=-k \frac{|u| u}{h^{\alpha}}-g h \frac{\partial z}{\partial x} .
\end{gathered}
$$

Then keeping smaller terms, and proceeding again as in [11, 12], a viscous Shallow Water system with new friction term of Darcy-Weisbach (resp. Manning) type is obtained 


$$
\begin{aligned}
\frac{\partial h}{\partial t}+\frac{\partial h u}{\partial x} & =0, \\
\frac{\partial h u}{\partial t}+\frac{\partial h u^{2}}{\partial x}+\frac{g}{2} \frac{\partial h^{2}}{\partial x} & =-k \frac{h^{-\alpha}|u| u}{\left(1+\frac{k}{3 v}|u| h^{1-\alpha}\right)^{2}}-g h \frac{\partial z}{\partial x}+4 v \frac{\partial}{\partial x}\left(h \frac{\partial u}{\partial x}\right)
\end{aligned}
$$

\section{Numerical Scheme}

In order to solve systems (3) and (4), we use FullSWOF_1D software which stands for Full Shallow Water equations for Overland Flow in 1 space Dimension [3, 15]. It is an open source $\mathrm{C}++$ codes, freely available from https://sourcesup.renater.fr/ projects/fullswof-1d/. It is distributed under CeCILL-V2 free software license. The structure of the code is made to facilitate the development of new evolutions. This software solves the shallow water Eq. (3) thanks to a well-balanced finite volume method based on the hydrostatic reconstruction $[16,17]$. This numerical method has good properties: water mass conservation, well-balanced property (at least preservation of lake at rest equilibrium) and positivity water height preservation. We have made some modifications in the sources to deal with system (4) as well. We will first give the main lines of the numerical method implemented in FullSWOF to solve system (3). Then we will explain the modification integrated in FullSWOF_ 1D to solve (4). In FullSWOF, the shallow water equations are solved thanks to a well-balanced finite volume scheme. The well-balanced property is achieved with the hydrostatic reconstruction, which is based on a general principle of reconstruction. We begin with a first order finite volume scheme for the homogeneous shallow water equations: choosing a positive and consistent numerical flux $F\left(U_{L}, U_{R}\right)$ (e.g. Rusanov, HLL, kinetic,... [2, 17]), it writes under the general form

$$
\frac{U_{i}^{\mathrm{star}}-U_{i}^{n}}{\Delta t}+\frac{F\left(U_{i}^{n}, \mathrm{U}_{i+1}^{n}\right)-F\left(U_{i-1}^{n}, \mathrm{U}_{i}^{n}\right)}{\Delta x}=0,
$$

where $\Delta \mathrm{t}$ is the time step and $\Delta \mathrm{x}$ is the space step. The idea is to modify this scheme by applying the numerical flux to the reconstructed variables. Reconstruction can be used to get higher order schemes (MUSCL, ENO, ...), and the higher order in time is obtained thanks to TVD-Runge-Kutta methods. The purpose of the hydrostatic reconstruction is to get a well-balanced scheme. It is designed to preserve at least steady states at rest $(u=0$ and $h+Z=C s t$ ). When it is directly applied on the initial scheme, it gives a first order scheme, while coupling it with high order reconstruction increases the order of the scheme and thus its accuracy.

We now give the implementation of this method to get high order accuracy. The first step consists in performing a high order reconstruction (MUSCL, ENO, ...). To deal properly with the topography source term $\partial_{x} z$, this reconstruction is applied on $u, h$ and $h+z$. Thus we get the set of reconstructed variables $\left(U_{m}, z_{m}\right)$ and $\left(U_{p}, z_{p}\right)$, on which the hydrostatic reconstruction is applied 


$$
\begin{aligned}
h_{\mathrm{i}+1 / 2 \mathrm{~L}} & =\max \left(h_{\mathrm{i}+1 / 2 \mathrm{~m}}+z_{\mathrm{i}+1 / 2 \mathrm{~m}}-\max \left(z_{\mathrm{i}+1 / 2 \mathrm{~m}}, z_{\mathrm{i}+1 / 2 \mathrm{p}}\right), 0\right), \\
U_{\mathrm{i}+1 / 2 \mathrm{~L}} & =\left(h_{\mathrm{i}+1 / 2 \mathrm{~L}}, h_{\mathrm{i}+1 / 2 \mathrm{~L}} u_{\mathrm{i}+1 / 2 \mathrm{~m}}\right), \\
h_{\mathrm{i}+1 / 2 \mathrm{R}} & =\max \left(h_{\mathrm{i}+1 / 2 \mathrm{p}}+z_{\mathrm{i}+1 / 2 \mathrm{p}}-\max \left(z_{i+1 / 2 \mathrm{~m}}, z_{\mathrm{i}+1 / 2 \mathrm{p}}\right), 0\right), \\
U_{\mathrm{i}+1 / 2 \mathrm{R}} & =\left(h_{\mathrm{i}+1 / 2 \mathrm{R}}, h_{\mathrm{i}+1 / 2 \mathrm{R}} u_{\mathrm{i}+1 / 2 \mathrm{p}}\right)
\end{aligned}
$$

The finite volume scheme (9) needs to be modified as follows

$$
\frac{U_{i}^{\mathrm{star}}-U_{i}^{n}}{\Delta t}+\frac{F_{\mathrm{i}+1 / 2 \mathrm{~L}}^{n}-F_{i-1 / 2 \mathrm{R}}^{n}-\mathrm{Fc}_{i}^{n}}{\Delta x}=0
$$

where

$$
\begin{aligned}
& F_{\mathrm{i}+1 / 2 \mathrm{~L}}^{n}=F_{\mathrm{i}+1 / 2}^{n}+S_{\mathrm{i}+1 / 2 \mathrm{~L}}^{n}, \\
& F_{\mathrm{i}-1 / 2 \mathrm{R}}^{n}=F_{\mathrm{i}-1 / 2}^{n}+S_{\mathrm{i}+1 / 2 \mathrm{R}}^{n},
\end{aligned}
$$

are left (resp. right) modifications of the numerical flux for the homogeneous system. In this formula, the numerical flux is now applied on the reconstructed variables $F_{\mathrm{i}+1 / 2}^{n}=F\left(U_{\mathrm{i}+1 / 2 \mathrm{~L}}^{n}, U_{\mathrm{i}+1 / 2 \mathrm{R}}^{n}\right)$ and we take

$$
S_{\mathrm{i}+1 / 2 \mathrm{~L}}^{n}=\left(\begin{array}{c}
0 \\
\frac{g h_{\mathrm{i}+1 / 2 \mathrm{~m}}^{2}-g h_{\mathrm{i}+1 / 2 \mathrm{~L}}^{2}}{2}
\end{array}\right) \quad, \quad S_{\mathrm{i}+1 / 2 \mathrm{R}}^{n}=\left(\begin{array}{c}
0 \\
\frac{g h_{i-1 / 2 \mathrm{p}}^{2}-g h_{i-1 / 2 \mathrm{R}}^{2}}{2}
\end{array}\right) .
$$

Finally, for consistency and well-balancing properties, a centered source term is added to the scheme

$$
\mathrm{Fc}_{i}^{n}=\left(\begin{array}{c}
0 \\
-g \frac{h_{i-1 / 2 \mathrm{p}}+h_{i-1 / 2 \mathrm{~m}}}{2}\left(z_{i+1 / 2 \mathrm{~m}}-z_{i-1 / 2 \mathrm{p}}\right)
\end{array}\right) .
$$

The chosen numerical strategy consists in the HLL flux (see [2, 17]) combined with a modified MUSCL reconstruction. It has shown to be the best compromise between accuracy, stability and CPU time cost [2]. The MUSCL reconstruction of a real variable $s$ writes

$$
s_{i-1 / 2 \mathrm{p}}=s_{i}-\Delta x \cdot \frac{D s_{i}}{2}, s_{\mathrm{i}+1 / 2 \mathrm{~m}}=s_{i}+\Delta x \cdot \frac{D s_{i}}{2}
$$

with the minmod slope limiter

$$
D \mathrm{~s}_{i}=\min \bmod \left(\frac{s_{i}-s_{i-1}}{\Delta x}, \frac{s_{\mathrm{i}+1}-s_{i}}{\Delta x}\right), \min \bmod (x, y)=\begin{array}{ll}
\min (x, y) & \text { if } x, y \geq 0 \\
\max (x, y) & \text { if } x, y \leq 0 \\
0 & \text { else }
\end{array}
$$


In order to keep the discharge conservation, the reconstruction of the velocity has to be modified as follows

$$
u_{i-1 / 2 \mathrm{p}}=u_{i}-\frac{h_{\mathrm{i}+1 / 2 \mathrm{~m}}}{h_{i}} \frac{\Delta x}{2} D u_{i}, u_{\mathrm{i}+1 / 2 \mathrm{~m}}=u_{i}-\frac{h_{\mathrm{i}-1 / 2 \mathrm{p}}}{h_{i}} \frac{\Delta x}{2} D u_{i} .
$$

We can notice that if we take $D s_{i}=0$, we recover the first order scheme in space. The friction term is treated numerically by a fractional step, with the following system

$$
\partial_{t} U=\left(\begin{array}{c}
0 \\
-g h S f
\end{array}\right)
$$

where $S f$ is the friction source term. This system is solved thanks to a semi-implicit treatment $[18,19]$. As an example, for the Manning friction law, it writes

$$
\begin{aligned}
h^{\mathrm{n}+1} & =h^{\mathrm{star}} \\
\frac{q^{\mathrm{n}+1}-q^{\mathrm{star}}}{\Delta t} & =-n^{2} \frac{q^{\mathrm{n}+1}\left|q^{n}\right|}{h^{n}\left(h^{\mathrm{n}+1}\right)^{4 / 3}} .
\end{aligned}
$$

This method allows to preserve stability (under a classical CFL condition) and steady states at rest. Finally a TVD-Runge Kutta (Heun) method is applied to get second order in time. This is what has been currently developed in FullSWOF to solve system (3). To solve our new system (4), we have integrated the new friction laws treated with a semi-implicit treatment such as (15). A Crank-Nicolson method is used to deal with the diffusion source term. The obtained tridiagonal linear system is solved thanks to the LAPACK library (Linear Algebra PACKage [20]). We have to mention that these modifications of the code are not currently available on FullSWOF website.

\section{Numerical Validations}

\subsection{Analytical Solutions}

FullSWOF has been widely validated on analytical solutions implemented in SWASHES library [21, 22]. Keeping this philosophy in mind, we have developed some analytical solutions to validate our approach following the main lines of [13]. From (4), steady state solutions are constructed. At stationary equilibrium this system reduces into 


$$
\begin{aligned}
q & =C s t \\
\frac{\partial Z}{\partial x} & =\frac{1}{g h}\left(\left(\frac{q^{2}}{h^{2}}-g h\right) \frac{\partial h}{\partial x}-\frac{k|q| q}{h^{\alpha+2}\left(1+\frac{k}{3 v}|q| h^{-\alpha}\right)^{2}}+4 v \frac{q}{h}\left(\frac{1}{h}\left(\frac{\partial h}{\partial x}\right)^{2}-\frac{\partial^{2} h}{\partial x^{2}}\right)\right)
\end{aligned}
$$

The above relation allows to compute topography corresponding to sufficiently smooth function for the free surface. For all the following tests, we will consider dry initial conditions $(h(t=0, x)=0 \mathrm{~m}$ and $u(t=0, x)=0 \mathrm{~m} / \mathrm{s})$, this allows to test the ability of the numerical to deal properly with wet/dry transitions and to catch steady state solutions. We will consider successively three kinds of regimes: subcritical (or fluvial), supercritical (or torrential) and transcritical, for both the new Manning and Darcy-Weisbach friction laws.

For each numerical result, we also compute the relative $L^{2}$ - error at the final time between the reference and the computed water height.

\subsection{Numerical Results}

\subsubsection{Subcritical Flow for Manning Friction Law}

A $1000 \mathrm{~m}$ long channel is considered. The following water height is chosen and plotted on Fig. 1:

$$
h_{e x}(x)=\left(\frac{4}{g}\right)^{1 / 3}\left(1+\frac{1}{2} \exp \left(-16\left(\frac{x}{1000}-\frac{1}{2}\right)^{2}\right)\right)
$$

Fig. 1 Comparison between the numerical result $Z+h$ (plotted every 50 points) and the reference solution $Z+h_{e x}$ for subcritical flow and Manning friction law

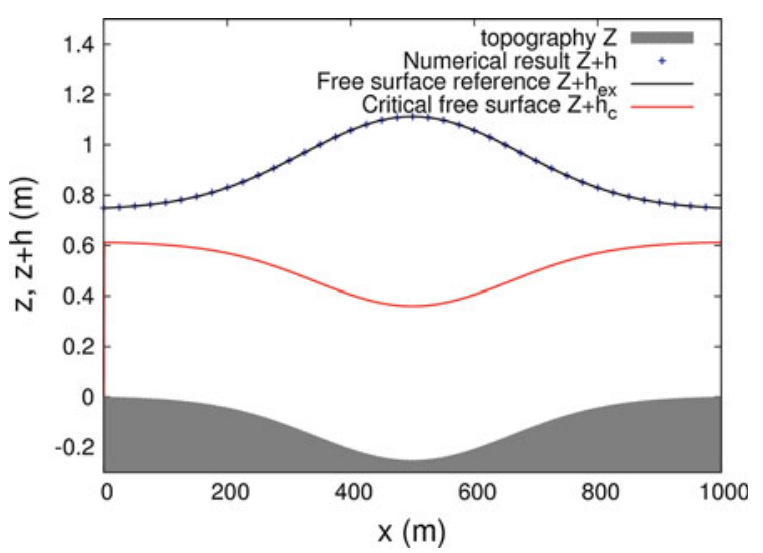


At the inflow boundary $x=0$, we impose a constant discharge $\mathrm{q}=1.5 \mathrm{~m}^{2} / \mathrm{s}$. At the outflow $x=1000 \mathrm{~m}$, we use the reference solution to impose the water height $h_{e x}(1000)$.

$$
\text { Parameters : } n=0.33, v=10^{-6}, \Delta x=0.5 \mathrm{~m}, \Delta t=0.02 \mathrm{~s} \text {. }
$$

We have represented the critical free surface $Z+h_{c}$ in red, where $h_{c}=\left(q^{2} / g\right)^{1 / 3}$ is the critical water height. This height allows to visualize the flow regime. We can notice that the subcritical stationary flow is perfectly catched by the numerical method (Fig. 1). The relative $L^{2}$ - error is approximately $5.6384 \times 10^{-5}$.

\subsubsection{Subcritical Flow for Darcy-Weisbach Friction Law}

A $1000 \mathrm{~m}$ long channel is considered. We consider the water height defined by (21). At the inflow boundary $x=0$, we impose a constant discharge $q=1.5 \mathrm{~m}^{2} / \mathrm{s}$. At the outflow $x=1000 \mathrm{~m}$, we use the reference solution to impose the water height $h_{e x}$ (1000) (Fig. 2).

$$
\text { Parameters : } f=0.25, v=10^{-6}, \Delta x=0.5 \mathrm{~m}, \Delta t=0.02 \mathrm{~s} \text {. }
$$

As for the Manning friction law (Sect. 4.2.1), we get a perfect agreement between the reference solution and the numerical solution. Moreover the relative $L^{2}$ - error is also approximately $5.6384 \times 10^{-5}$.

\subsubsection{Supercritical Flow for Manning Friction Law}

A $1000 \mathrm{~m}$ long channel is considered. The following water height is chosen and plotted on Fig. 3:

Fig. 2 Comparison between the numerical result $Z+h$ (plotted every 50 points) and the reference solution $Z+h_{e x}$ for subcritical flow and Darcy-Weisbach friction law

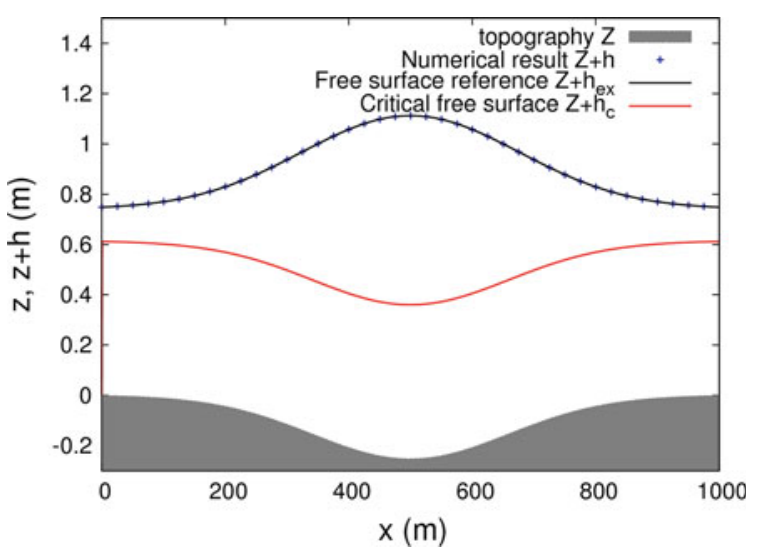


Fig. 3 Comparison between the numerical result $Z+h$ (plotted every 50 points) and the reference solution $Z+h_{e x}$ for supercritical flow and Manning friction law

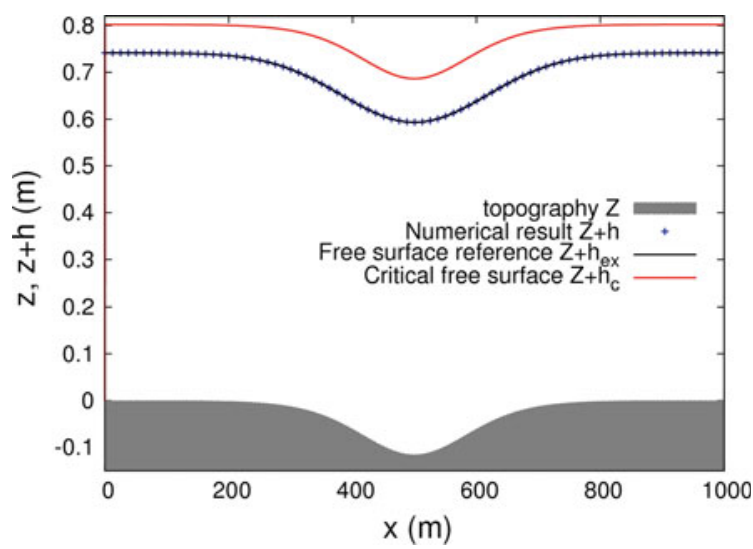

$$
h_{e x}(x)=\left(\frac{4}{g}\right)^{1 / 3}\left(1-\frac{1}{5} \exp \left(-36\left(\frac{x}{1000}-\frac{1}{2}\right)^{2}\right)\right)
$$

At the inflow boundary, we impose a constant discharge $q=2.25 \mathrm{~m}^{2} / \mathrm{s}$ and a constant water height equal to the steady state reference solution at inflow $h_{e x}(0)$. The outflow boundary at $x=1000 \mathrm{~m}$ is let free (Neumann condition).

$$
\text { Parameters : } n=0.33, v=10^{-6}, \Delta x=0.25 \mathrm{~m}, \Delta t=0.02 \mathrm{~s} \text {. }
$$

On Fig. 3, we can notice that the numerical method allows to catch perfectly the torrential equilibrium for the Manning friction law. For this case, the relative $L^{2}$ error is approximately $1.4876 \times 10^{-5}$.

\subsubsection{Supercritical Flow for Darcy-Weisbach Friction Law}

A $1000 \mathrm{~m}$ long channel is considered. We consider the water height defined by formula (22). At the inflow boundary $x=0$, we impose a constant discharge $q=2.25 \mathrm{~m}^{2} / \mathrm{s}$ and a constant water height equal to the steady state reference solution at the income $h_{e x}(0)$. The outflow boundary at $x=1000 \mathrm{~m}$ is let free (Neumann condition).

$$
\text { Parameters : } f=0.25, v=10^{-6}, \Delta x=0.25 \mathrm{~m}, \Delta t=0.02 \mathrm{~s} \text {. }
$$

Perfect agreement is observed between the reference solution and the numerical solution (Fig. 4) and the relative $L^{2}$-error is approximately $1.5 .27 \times 10^{-5}$. 
Fig. 4 Comparison between the numerical result $Z+h$ (plotted every 50 points) and the reference solution $Z+h_{e x}$ for supercritical flow and Darcy-Weisbach friction law

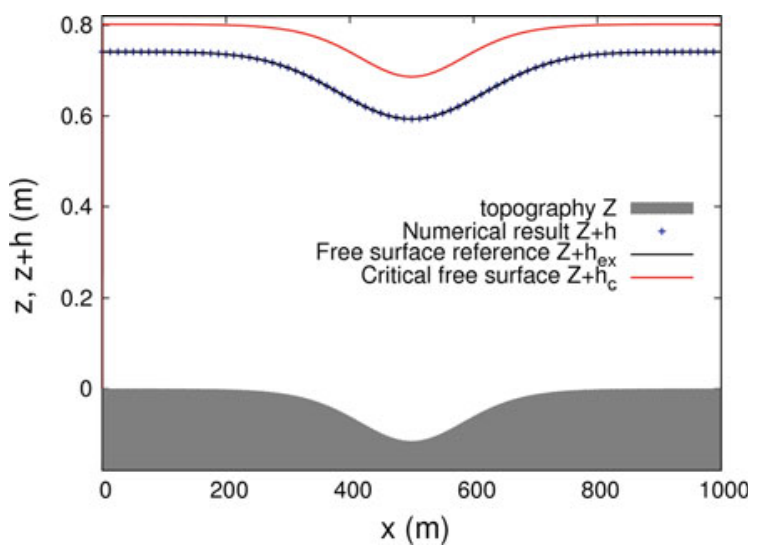

\subsubsection{Transcritical Flow for Manning Friction Law}

A $100 \mathrm{~m}$ long channel is considered. The following water height is chosen and plotted on Fig. 5:

$$
h_{e x}(x)=\frac{1}{2} \cos \left(\frac{\pi}{100} x\right)+1
$$

At the inflow boundary $x=0$, we impose a constant discharge $q=2.2 \mathrm{~m}^{2} / \mathrm{s}$. The outflow boundary at $x=100 \mathrm{~m}$ is let free (Neumann condition).

$$
\text { Parameters : } n=0.33, v=10^{-6}, \Delta x=0.5 \mathrm{~m}, \Delta t=0.01 \mathrm{~s} \text {. }
$$

Transcritical solutions might be difficult to catch numerically. We can see on Fig. 5, that this numerical difficulty is overcome by the scheme we have considered.

Fig. 5 Comparison between the numerical result $Z+h$ (plotted every 5 points) and the reference solution $Z+h_{e x}$ for transcritical flow and Manning friction law

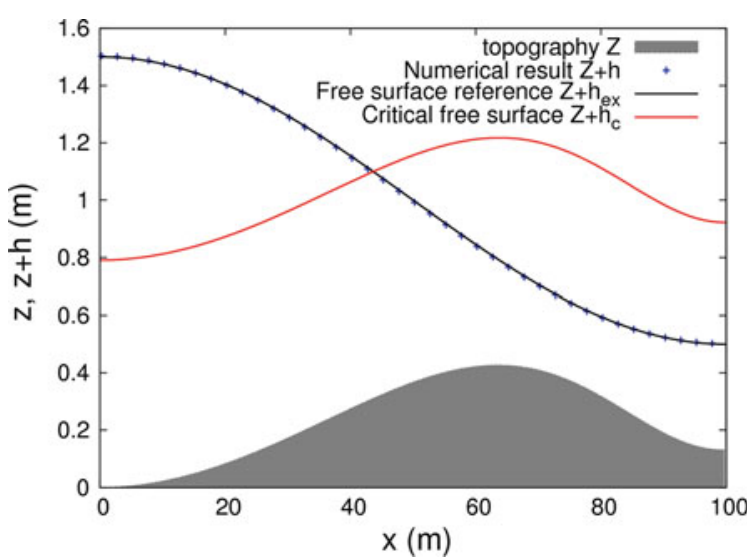


Fig. 6 Comparison between the numerical result $Z+h$ (plotted every 5 points) and the reference solution $Z+h_{e x}$ for transcritical flow and Darcy-Weisbach friction law

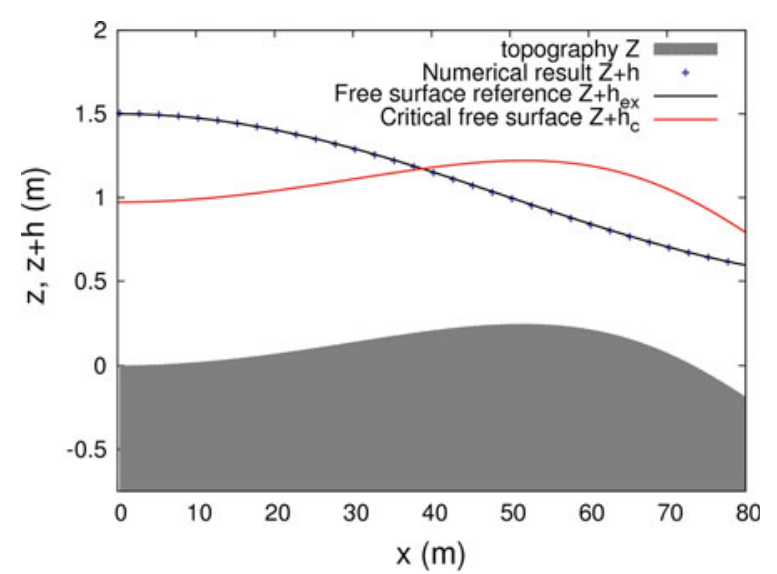

The equilibrium is perfectly obtained by the numerical method. The relative $L^{2}$ error is approximately $1.208 \times 10^{-3}$.

\subsubsection{Transcritical Flow for Darcy-Weisbach Friction Law}

A $80 \mathrm{~m}$ long channel is considered. We consider the water height defined by (23). At the inflow boundary $x=0$, we impose a constant discharge $\mathrm{q}=3 \mathrm{~m}^{2} / \mathrm{s}$. The outflow boundary at $x=80 \mathrm{~m}$ is let free (Neumann condition).

$$
\text { Parameters : } f=0.25, v=10^{-6}, \Delta x=0.5 \mathrm{~m}, \Delta t=0.01 \mathrm{~s} \text {. }
$$

As for the Manning friction law (see Sect. 4.2.5), the transcritical steady state is perfectly reached by the numerical (as observed on Fig. 6) and the relative $L^{2}$ - error is approximately $1.2885 \times 10^{-3}$.

We have validated our numerical method on various flow regimes. To complete this work, some convergence analysis and accuracy study need to be done. This will be considered in forthcoming work. We have to validate our model, this will be done in next section on real data.

\section{First Attempt on Real Data}

In this section, we aim at validating our model (4) on real data. For this case, an experimental flow over an inclined corrugated bottom (Fig. 7) is considered with an inflow discharge of $q=3.9 \times 10^{-4} \mathrm{~m}^{2} / \mathrm{s}$. Free surface profile is measured at steady state along a $55 \mathrm{~cm}$ long profile with a $0.5 \mathrm{~mm}$ resolution using the device described in [23]. A succession of transcritical transitions and hydraulic jumps is obtained. 


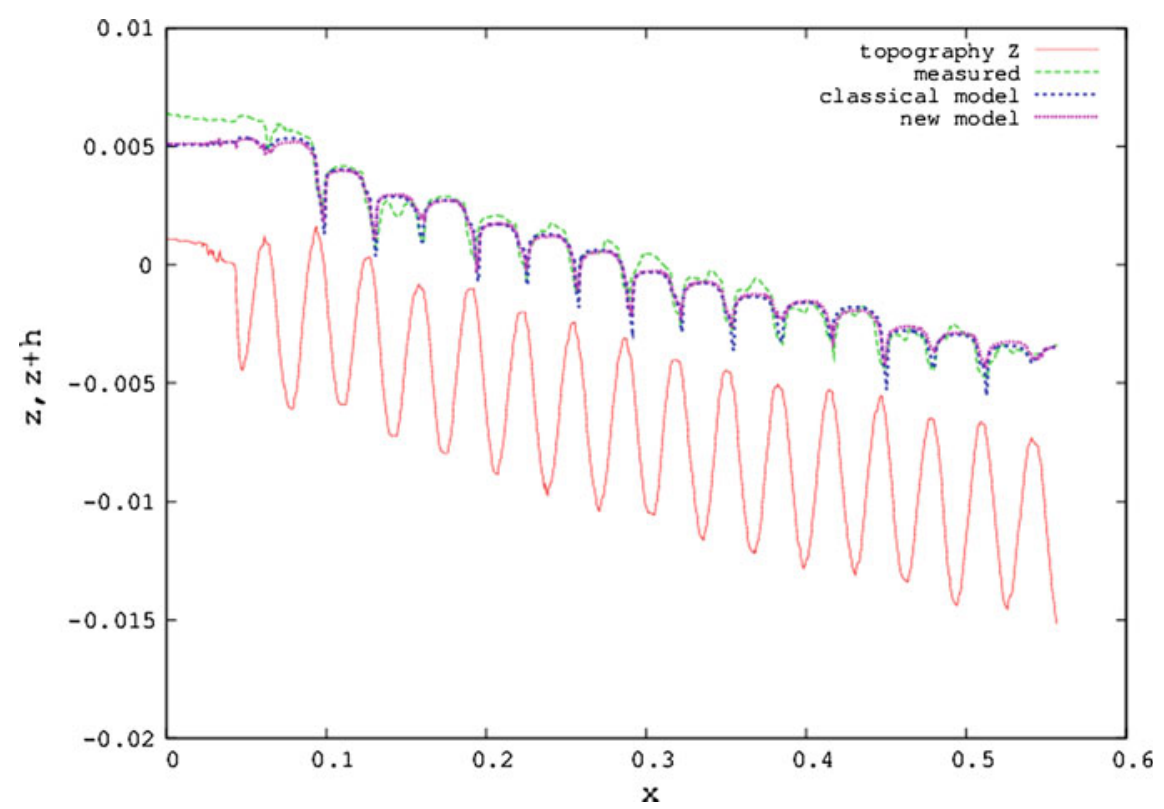

Fig. 7 Comparison between the two models against experimental data over a corrugated bottom

FullSWOF_1D has already been run on this data set [15]. It has been able to locate the hydraulic jumps correctly but the simulated solution has exhibited shocks steeper than the measured ones. In this work, we have considered successively system (3) and system (4) with Manning friction law. The length of the domain is $L=0.557 \mathrm{~m}$, the space step length is $\Delta x=5 \times 10^{-4} \mathrm{~m}$. For system (4), the viscosity is $v=2 \times 10^{-5}$.

On Fig. 7, we can notice that the hydraulic jumps are less steep with the new model (4) than with the classical model. The relative $L^{2}$ - error on the water height between the experimental and the classical shallow water model is approximately $9.5872 \times 10^{-2}$ and is closed to the one between the experimental and the new model which is approximately $9.4747 \times 10^{-2}$. More comparisons on real data will be necessary to completely validate this new model.

\section{Conclusion and Perspectives}

In this work, we have developed a new shallow-water system with diffusion source term and new Manning and Darcy-Weisbach friction laws. We have validated a numerical method adapted to this system on new various analytical solutions. This numerical method showed good agreement with the reference solutions. In forthcoming work, we will have to do convergence analysis. Then we have applied this 
model on real data. This model seems to be more adapted than the classical shallow-water model. We will have to try other flow regimes to validate completely this new model.

Acknowledgements The authors whish to thanks the ANR-11-JS01-006-01 project CoToCoLa (Contemporary Topics on Conservation Laws), Carine Lucas for her advices and Frédéric Darboux for the data used in Sect. 4.2.

\section{References}

1. de Saint-Venant, A. J.-C. (1871). Théorie du mouvement non-permanent des eaux, avec application aux crues des rivières et à l'introduction des marées dans leur lit. Comptes Rendus de l'Académie des Sciences, 73, 147-154.

2. Delestre, O. (2010). Simulation du ruissellement d'eau de pluie sur des surfaces agricoles. $\mathrm{PhD}$ thesis University of Orléans, in french. http://tel.archives-ouvertes.fr/INSMI/tel-00531377/fr.

3. Delestre, O., Cordier, S., Darboux, F., Du, M., James, F., \& Laguerre, C., et al. (2014). FullSWOF: A software for overland flow simulation. In P.Gourbesville, J. Cunge, \& G. Caignaert, (Eds.), Advances in Hydroinformatics, Springer Hydrogeology (pp. 221-231). Springer: Singapore.

4. Esteves, M., Faucher, X., Galle, S., \& Vauclin, M. (2000). Overland flow and infiltration modelling for small plots during unsteady rain: numerical results versus observed values. Journal of Hydrology, 228, 265-282.

5. Tatard, L., Planchon, O., Wainwright, J., Nord, G., Favis-Mortlock, D., Silvera, N., et al. (2008). Measurement and modelling of high-resolution flow-velocity data under simulated rainfall on a low-slope sandy soil. Journal of Hydrology, 348(1-2), 1-12.

6. Goutal, N., \& Maurel, F. (2002). A finite volume solver for 1D shallow-water equations applied to an actual river. International Journal for Numerical Methods in Fluids, 38, 1-19.

7. Caleffi, V., Valiani, A., \& Zanni, A. (2003). Finite volume method for simulating extreme flood events in natural flood events in natural channels. Journal of Hydraulic Research, 41(2), 167-177.

8. Alcrudo, F., \& Gil, E. (1999). The Malpasset dam break case study. Proceedings of the 4th CADAM Workshop, Zaragoza (pp. 95-109).

9. Valiani, A., Caleffi, V., \& Zanni, A. (2002). Case study: Malpasset dam-break simulation using a two-dimensional finite volume methods. Journal of Hydraulic Engineering, 128(5), 460-472.

10. Popinet, S. (2011). Quadtree-adaptive tsunami modelling. Ocean Dynamics, 61(9), 12611285.

11. Gerbeau, J.-F., \& Perthame, B. (2001). Derivation of viscous Saint-Venant system for laminar shallow water; numerical validation. Discrete and Continuous Dynamical Systems-Series S, $1,89-102$.

12. Marche, F. (2007). Derivation of a new two-dimensional viscous shallow water model with varying topography, bottom friction and capillary effects. European Journal of Mechanics B/Fluids, 26, 49-63.

13. MacDonald, I., Baines, M. J., Nichols, N. K., \& Samuels, P. G. (1997). Journal of Hydraulic Engineering, 123, 1041-1045.

14. Chow, V. T. (1959). Open-channel hydraulics. New York: McGraw-Hill.

15. Delestre, O., Darboux, F., James, F., Lucas, C., Laguerre, C., \& Cordier, S. (Submitted). FullSWOF: A free software package for the simulation of shallow water flows. arxiv. org/abs/1401.4125. 
16. Audusse, E., Bouchut, F., Bristeau, M.-O., Klein, R., \& Perthame, B. (2004). A fast and stable well-balanced scheme with hydrostatic reconstruction for shallow water flows. Journal of Scientific Computing, 25(6), 2050-2065.

17. Bouchut, F. (2004). Nonlinear stability of finite volume methods for hyperbolic conservation laws, and well-balanced schemes for sources. Frontiers in Mathematics. Basel: Birkhauser.

18. Bristeau, M.-O., \& Coussin, B. (2001). Boundary conditions for the shallow water equations solved by kinetic schemes. Inria report RR-4282.

19. Fiedler, R. F., \& Ramirez, J. A. (2000). A numerical method for simulating discontinuous shallow flow over an infiltrating surface. International Journal for Numerical Methods in Fluids, 32, 219-240.

20. Anderson, E., Bai, Z., Bischof, C., Blackford, L.S., Demmel, J., \& Dongarra, J., et al. (1999). LAPACK Users' guide ( ${ }^{\text {rd }}$ ed.). Philadelphia: Society for Industrial and Applied Mathematics.

21. Delestre, O., Lucas, C., Ksinant, P.-A., Darboux, F., Laguerre, C., Vo, T. N. T., et al. (2013). SWASHES: A compilation of Shallow-Water analytic solutions for hydraulic and environmental studies. International Journal for Numerical Methods in Fluids, 72, 269-300. doi:10.1002/fld.3741.

22. Delestre, O., Lucas, C., Ksinant, P.-A., Darboux, F., Laguerre, C., \& James, F., et al. (2014). SWASHES: A library for benchmarking in hydraulic. In p. Gourbesville, J. Cunge \& G. Caignaert, (Eds.), Advances in Hydroinformatics, Springer Hydrogeology (pp. 233-243). Springer: Singapore.

23. Legout, C., Darboux, F., Nédélec, Y., Hauet, A., Esteves, M., Renaux, B., et al. (2012). High spatial resolution mapping of surface velocities and depths for shallow overland flow. Earth Surface Processes and Landforms, 37(9), 984-993. doi:10.1002/esp.3220. 